\section{Bioethanol als Kraftstoff - Stand und Perspektiven}

\author{
von Norbert Schmitz, Meó Consulting Team
}

Dieser Beitrag gibt einen Überblick zur Bereitstellung und Verwendung von Bioethanol als Kraftstoff. Ausgehend von der Ethanolerzeugung in Deutschland wird auf die hierfür nötige Rohstoffversorgung und -eignung eingegangen, um dann einen Ausblick auf das potenzielle Bioethanol-Produktionsvolumen zu geben. Die Verhältnisse in Brasilien - als weltweit kostengünstigster Ethanolproduzent - werden als „Benchmark“ für die europäische Ethanolproduktion herangezogen und erläutert. Abschließend konzentrieren sich die Ausführungen auf Energieund Treibhausgasbilanzen bei der Ethanolproduktion. Hierbei sollen die immer wieder auftretenden Behauptungen entkräftet werden, die Bioethanolerzeugung würde negative Energiebilanzen aufweisen. Dabei wird auf die in den vergangenen Jahren kontinuierlichen Verbesserungen entlang der Wertschöpfungskette und auf innovative, neue Verfahren eingegangen.

\section{Einleitung}

Der Transportsektor trägt wesentlich $\mathrm{zu}$ den $\mathrm{CO}_{2}$-Emissionen bei und spielt deshalb bei der beabsichtigten Verminderung der Emissionen um $8 \%$ gegenüber 1990 für die Periode 2008 bis 2012 in der EU eine besondere Rolle. Die Bundesregierung hat sich im Rahmen des Klimaschutzprogramms zu Minderungen der Treibhausgase verpflichtet. Die verstärkte Nutzung regenerativer Energiequellen kann hierzu einen Beitrag leisten. Während für stationäre Energieanlagen eine Reihe von regenerativen Energiequellen eingesetzt werden können, sind die Möglichkeiten im Transportsektor sehr begrenzt. Ethanol bzw. ETBE ${ }^{1}$ für Ottomotoren sowie Biodiesel (RME) bzw. reines Pflanzenöl für Dieselmotoren sind derzeit die einzigen technisch realisierbaren und marktfähigen erneuerbaren Kraftstoffe. ETBE wird bereits in Deutschland eingesetzt und die direkte Beimischung von Bioethanol zum Kraftstoff erfolgt regional. Die Mineralölindustrie bereitet zudem den Einsatz weiterer Ether wie TAEE ${ }^{2}$ vor; entsprechende Verarbeitungsanlagen sollen an mehreren Raffineriestandorten errichtet werden. Biogas spielt bislang im deutschen Markt keine
Rolle. Biokraftstoffe der zweiten Generation (z. B. Fischer-Tropsch-Kraftstoff) werden kurzund mittelfristig ebenfalls keine wesentlichen Marktanteile verbuchen können (vgl. IEA 2004; Quirin et al. 2004).

Im März 2003 hat das Europäische Parlament eine Richtlinie verabschiedet, wonach bis zum Jahr 2005 biogene Kraftstoffe einen Anteil von mindestens $2 \%$ des gesamten Kraftstoffverbrauchs erreichen sollen. Für das Jahr 2010 strebt die EU einen Wert von $5,75 \%$ an. $^{3}$ In Deutschland hat der Gesetzgeber mit der Änderung des Mineralölsteuergesetzes die Befreiung der Biokraftstoffe von der Mineralölsteuer beschlossen.

$\mathrm{Zu}$ Irritationen hat die im Koalitionsvertrag vom 11.11.2005 formulierte Ankündigung geführt, die Befreiung der Biokraftstoffe von der Mineralölsteuer durch einen Beimischungszwang zu ersetzen. Dies würde in der Konsequenz zu einem verstärkten Importdruck und zu einer deutlichen Schwächung der Marktposition heimischer Biokraftstofferzeuger führen. Wie auch immer die zukünftigen Regelungen für die Förderung von Biokraftstoffen ausgelegt werden: Es ist grundsätzlich davon auszugehen, dass Bioethanol eine wesentliche Rolle im Markt spielen wird (vgl. BMVEL 2005; FNR 2005). Die von der EU-Kommission geforderte jährliche Überprüfung der Höhe der steuerlichen Förderung zur Verhinderung einer möglichen Überkompensation führt ebenfalls nicht zu stabilen Rahmenbedingungen für Investoren, weder in der Biokraftstoff- noch in der Mineralölindustrie (vgl. Deutscher Bundestag 2005).

Vielfältige Ziele werden mit der steuerlichen Förderung von Bioethanol und anderen Biokraftstoffen verfolgt, wie Tab. 1 (siehe nächste Seite) zeigt.

\section{Bioethanol in Deutschland}

Die Größe des zukünftigen Bioethanolmarktes hängt davon $\mathrm{ab}$, ob und wie viel Bioethanol dem Ottokraftstoff direkt beigemischt und in welchem Volumen ETBE hergestellt wird bzw. auch inwieweit andere Verwendungsoptionen wie TAEE, FAEE $^{4}$ oder Beimischungen in Diesel realisiert werden. Die Mineralölindustrie favorisiert die Verwendung von Ethern im Kraftstoff. Bedenken, die in den USA zum Verbot von MTBE (Methyl-tertiär-Butylether) 
Tab. 1: Begründung für die Förderung von Bioethanol

\begin{tabular}{|c|c|}
\hline Klimapolitik & $\begin{array}{l}\text { Die EU hat sich zur Reduzierung der Treibhausgasemissionen verpflichtet. Der Trans- } \\
\text { portsektor trägt wesentlich zu den } \mathrm{CO}_{2} \text {-Emissionen bei und spielt deshalb bei der beab- } \\
\text { sichtigten Verminderung der Emissionen um } 8 \% \text { gegenüber } 1990 \text { für die Zielperiode } \\
2008 \text { bis } 2012 \text { in der EU eine besondere Rolle. Bioethanol als Substitut von Ottokraft- } \\
\text { stoff trägt wesentlich zur Reduzierung von } \mathrm{CO}_{2} \text {-Emissionen bei. }\end{array}$ \\
\hline Energiepolitik & $\begin{array}{l}\text { - Die Energiepolitik will einerseits die Abhängigkeit von fossilen Rohstoffen, andererseits } \\
\text { die Importabhängigkeit reduzieren. } \\
\text { - Bioethanol kann zu beiden Zielen Beiträge leisten. }\end{array}$ \\
\hline Agrarpolitik & $\begin{array}{l}\text { - Die Agrarpolitik sucht, insbesondere nach der Osterweiterung der EU und aufgrund der } \\
\text { aktuellen WTO-Verhandlungen, Beschäftigungs- und Einkommensalternativen für die } \\
\text { Landwirtschaft sowie Perspektiven für ländliche Gebiete. } \\
\text { - Die heimische Landwirtschaft kann u. a. Rohstoffe für die Bioethanolproduktion liefern. }\end{array}$ \\
\hline Handelspolitik & $\begin{array}{l}\text { - Die landwirtschaftliche Überschussproduktion soll nicht mehr subventioniert im Ausland } \\
\text { abgesetzt werden. Importabhängigkeiten sollen reduziert werden. Neue Handelsvereinba- } \\
\text { rungen sind in Vorbereitung. } \\
\text { - Bioethanol bietet die Chance zur Etablierung neuer Handelspartnerschaften, z. B. mit } \\
\text { Mercosur / Brasilien. }\end{array}$ \\
\hline
\end{tabular}

geführt haben, werden in Deutschland offensichtlich nicht geteilt.

Der Absatz von Ottokraftstoff in der Europäischen Union (EU-25) beträgt derzeit knapp 120 Mio. $t$ pro Jahr. Die fünf großen Ottokraftstoffmärkte sind Deutschland, Großbritannien, Italien, Frankreich und Spanien. In diesen fünf Ländern werden mit 83 Mio. $t$ rund zwei Drittel des gesamten Ottokraftstoffs verbraucht. Insbesondere in Deutschland zeigt sich - als Folge steigender Dieselfahrzeug-Zulassungen, sinkenden Verbrauchs der Fahrzeuge und rückläufiger Kilometerleistungen - ein deutlicher Rückgang des Ottokraftstoffverbrauchs. Während der Verbrauch im Jahr 2000 noch bei 28,8 Mio. t lag, schrumpfte er bis zum Jahr 2005 auf 23,5 Mio. t. Für das Jahr 2010 wird ein Verbrauch von 22,9 Mio. t Ottokraftstoff vorhergesagt, für das Jahr 2020 von nur noch 17,5 Mio. t.

Bei der derzeit geltenden Kraftstoffnorm kann dem Ottokraftstoff direkt bis zu 5 Vol.-\% Bioethanol beigemischt werden. Eine $5 \%$ ige Beimischung führt in Deutschland zu einem Marktpotential von etwa 1,7 Mio. $\mathrm{m}^{3}$ Bioethanol. ${ }^{5}$ Zum Vergleich: Derzeit beträgt in der EU-25 die Ethanolproduktionskapazität 3,1 Mio. $\mathrm{m}^{3}$. Die direkte Beimischung erfordert die Lösung der sog. „Dampfdruckproblematik“6 sowie eine abgestimmte Vorgehensweise der Mineralölindustrie. Ethanol ist ein Lösungsmittel und im Gegensatz zu Ottokraftstoff mit Wasser völlig mischbar. Diese Eigenschaft von
Ethanol kann zu Problemen führen, wenn Ottokraftstoffe mit Bioethanolanteil mit Wasser in Berührung kommen. ${ }^{7}$

Im Vergleich zur direkten Beimischung hat ETBE den großen Vorteil, dass es aus technischer Sicht unproblematisch und in der Automobil- wie Mineralölindustrie allgemein akzeptiert ist. In der Vergangenheit wurden in den Raffinerien zur Verbesserung der Klopffestigkeit von Ottokraftstoffen hauptsächlich Bleialkyle eingesetzt. Die Herausnahme des Bleis und die zunehmende Auslegung moderner Motoren auf Superbedarf erfordern heute andere Verfahren zur Bereitstellung hochklopffester Kraftstoffe. Die Klopffestigkeit wird durch die Zugabe von Ethern verbessert. Ether sind sauerstoffhaltige Kohlenwasserstoffverbindungen, die sich $u$. a. durch hohe Oktanzahlen und niedrigen Dampfdruck auszeichnen. Die hohe Oktanzahl macht diese Komponenten für das Aufmischen hochoktaniger Ottokraftstoffe wertvoll. Die Herstellung von Ethern erfolgt durch Reaktion von Alkoholen mit verzweigten Olefinen.

Die MTBE- und ETBE-Kapazitäten betragen insgesamt 5,2 Mio. $t$ in der EU-25. MTBE wird aus Methanol und Isobuten hergestellt. Will man statt MTBE ETBE herstellen, wird das Methanol durch Ethanol ersetzt. An den technischen Anlagen sind nur relativ geringe Änderungen vorzunehmen. Seit Anfang 2004 wird in Deutschland ETBE hergestellt. Die Umstellungen an den Produktionsstandorten laufen, so 
dass seit Ende 2005 in Deutschland überwiegend ETBE hergestellt wird.

Im Jahr 2003 wurden in Deutschland etwa $280.000 \mathrm{~m}^{3}$ Ethanol erzeugt, in 2004 war das Erzeugungsvolumen etwas niedriger. Dieses Volumen macht etwa $10 \%$ der EU-Produktion aus. Weltweit wurden 2004 etwa 41 Mio. m ${ }^{3}$ Ethanol hergestellt - überwiegend in Amerika (vgl. Kap. 4).

Der deutsche Ethanolsektor ist geprägt durch das Branntweinmonopol sowie kleine und mittelgroße Erzeuger von Agraralkohol und der zweitgrößten europäischen SyntheseEthanol-Anlage. Traditionell spielt die deutsche Ethanolindustrie aufgrund des konservierenden Charakters des Branntweinmonopols im europäischen Kontext nur eine untergeordnete Rolle. Deutschland ist der wichtigste EthanolImportmarkt der EU. Die Importe nehmen seit geraumer Zeit zu und setzen die heimischen Produzenten zunehmend unter Druck. Die Exporte Deutschlands betragen dagegen nur ein Drittel des Importvolumens und sind zudem schrumpfend. Bei den Exporten handelt es sich zudem überwiegend um Syntheseethanol.
Wichtiger als die bestehenden Anlagen für die Entwicklung des Angebots von Bioethanol sind die neuen Großanlagen. Die beiden Unternehmen Südzucker und Sauter haben drei Anlagen mit einer Gesamtkapazität von rund $540.000 \mathrm{~m}^{3}$ in den neuen Bundesländern errichtet. Neben diesen Großanlagen sind auch zahlreiche weitere Anlagen unterschiedlicher Größenordnung im Bau bzw. in der Planung. Die Gesamterzeugungskapazität in Deutschland betrug damit Ende 2005 etwa $900.000 \mathrm{~m}^{3}$. Die Kapazität der Großanlagen ist mehr als ausreichend, um die Nachfrage nach Bioethanol für die ETBE-Produktion zu decken (ca. $360.000 \mathrm{~m}^{3}$ bei einer angenommenen Jahresproduktion von $600.000 \mathrm{t}$ in Deutschland). Ausreichenden Absatz im heimischen Markt für diese Anlagen wird es nur bei der direkten Beimischung zum Ottokraftstoff geben. Das bestehende Überangebot wird wachsen, wenn weitere Anlagen gebaut werden. ${ }^{8}$

Die nachfolgende Abb. 1 zeigt den Produktionsablauf in einer großen Bioethanolanlage, die aus Gerste oder Weizen über die wesentlichen Produktionsschritte Rohstoffaufbe-

\section{Abb. 1: Typisches Ablaufschema einer Getreide verarbeitenden Ethanolfabrik}

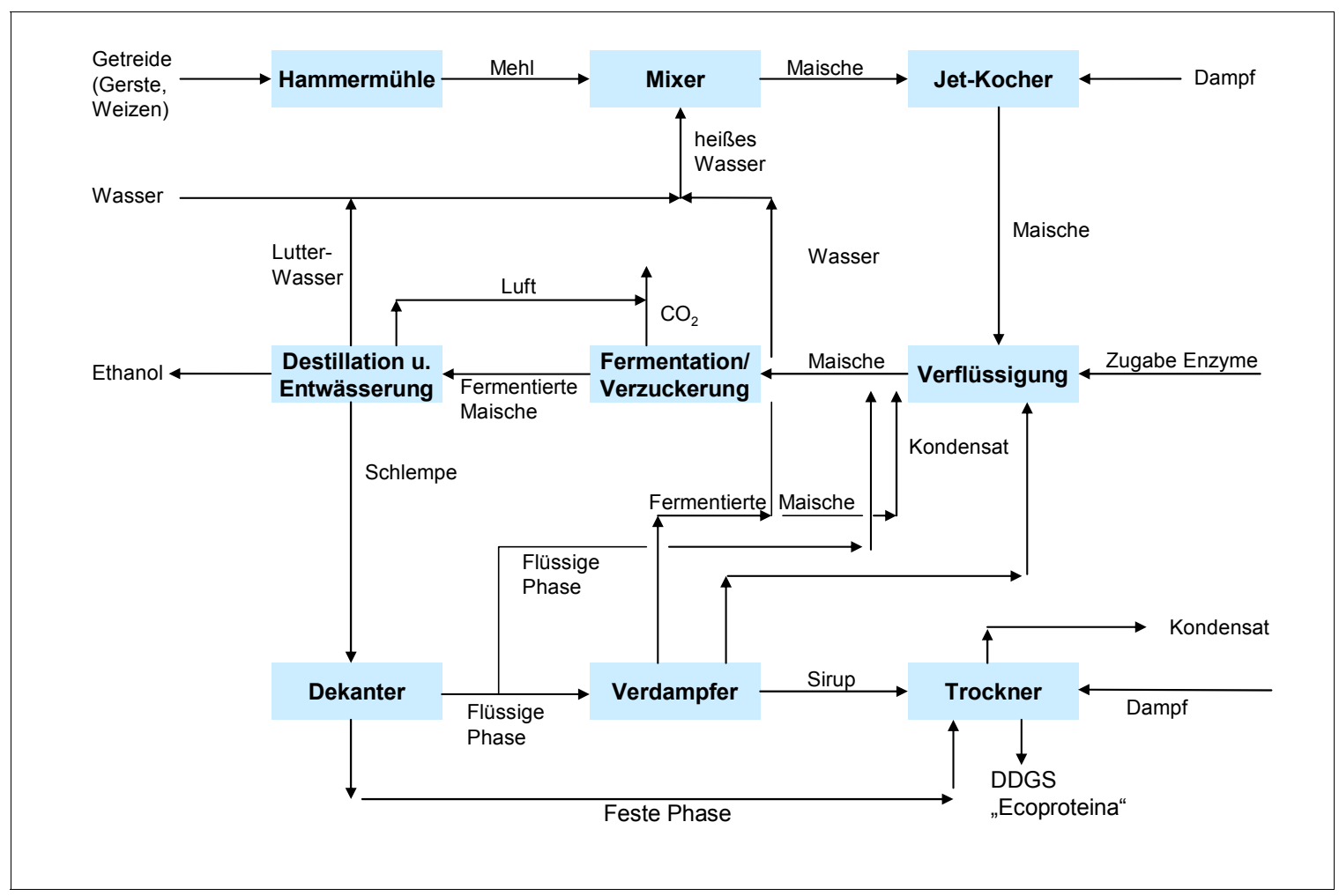

Quelle: Schmitz 2005 
reitung, Fermentation und Destillation Ethanol sowie ein eiweißreiches Futtermittel (DDGS) ${ }^{9}$ herstellt. Das bei der Fermentation freiwerdende $\mathrm{CO}_{2}$ kann ebenfalls vermarktet werden, beispielsweise an die Getränkeindustrie.

Die internationale Wettbewerbsfähigkeit der deutschen Bioethanolerzeugung kann durch die Nutzung der Möglichkeiten des novellierten Erneuerbare-Energien-Gesetz (EEG) erheblich verbessert werden. Voraussetzung für die Einspeisevergütung ist die Erzeugung von Biogas und dessen Verstromung in einer KraftWärme-Kopplungs-Anlage. In landwirtschaftlichen Brennereien ist dies aufgrund der relativ kleinen Kapazitäten bereits heute mit bewährter Technik möglich. Bei Bioethanolanlagen im industriellen Maßstab ist dies mit herkömmlicher Technik bislang nicht möglich und erfordert innovative Verfahren.

$\mathrm{Ob}$ es zu einem weiteren Ausbau der deutschen Ethanolproduktionskapazitäten kommen wird, hängt u. a. vom Ergebnis der WTO- und der EU-Mercosur-Verhandlungen ab. Unsichere Rahmenbedingungen (insbesondere hinsichtlich des Zollschutzes) führen dazu, dass schon geplante Investitionsvorhaben zunächst zurückgestellt werden. Auch die vorgeschlagene Regelung im Koalitionsvertrag der derzeitigen Regierungsparteien führt zu einer Verunsicherung möglicher Investoren.

Wettbewerbsintensität und Preisniveau im europäischen Bioethanolmarkt werden primär ein Resultat von politischen Entscheidungen sein. Eine Reduzierung des Außenschutzes und die Einräumung von Importkontingenten wird Druck auf die Ethanolpreise ausüben. Europäische Produzenten können mit Produzenten aus Brasilien nicht konkurrieren. Im freien Wettbewerb würden sie aus dem Markt ausscheiden und ein weiterer Ausbau der europäischen Bioethanolindustrie würde nicht stattfinden. Hohe Ethanolpreise im Markt stellen sich dann ein, wenn die Nachfrage schneller wächst als das Angebot.

Der wachsende Dieselmarkt ist bislang hinsichtlich einer Bioethanol-Beimischung von den Marktteilnehmern nicht in Erwägung gezogen worden. Unter Verwendung entsprechender Additive ist aber auch „Diesohol“ eine mögliche Option, die den Absatzmarkt für Bioethanol beträchtlich anwachsen lässt. Allerdings lässt sich damit nicht das Problem lösen, dass bereits bei geringfügigen Beimischungen von Ethanol der Flammpunkt deutlich abgesenkt würde. Dies ist nach der Diesel-Norm nicht zulässig.

Ein weiterer Wachstumstreiber wäre die Einführung von Flexible Fuel Vehicles $(\mathrm{FFVs})^{10}$. Erste Initiativen zur Markteinführung wurden gestartet. FFVs ermöglichen dem Verbraucher die bewusste Entscheidung für die Verwendung eines überwiegend regenerativen Kraftstoffs. FFVs erhöhen zudem die „Visibility“ von Bioethanol im Markt. Allerdings ist der Erfolg dieser Option auch von der Fortführung der Steuerbefreiung von Biokraftstoffen abhängig.

In Deutschland wurde die Steuerbefreiung von Biokraftstoffen vom Gesetzgeber mit ökologischen, energie-, beschäftigungs- und strukturpolitischen Argumenten begründet. Auch sollte die Steuerbefreiung Innovationen fördern. Sofern es aufgrund von steigenden Importen nicht zu einer Ausweitung der Produktion in Deutschland kommt, werden energie-, beschäftigungs- und strukturpolitische Ziele weitgehend nicht erreicht.

\section{Rohstoffe für die Ethanolerzeugung in Deutschland}

Ethanol kann in den erforderlichen Mengen für den Bioethanolmarkt nur aus den landwirtschaftlichen Rohstoffen hergestellt werden, bei denen ein Mindestmaß an Anbauerfahrung und eine möglichst bestehende bzw. nahe liegende großtechnische Nutzung vorhanden sind. Bei Zuckerrüben, Weizen, Roggen, Triticale (eine Kreuzung von Weizen und Roggen, Anmerkung der Redaktion), Körnermais und Kartoffeln verfügt die deutsche Landwirtschaft über erhebliche Anbauerfahrung und erzielt hohe Erträge meist in Verbindung mit einer hohen Ertragssicherheit. Unter den gegebenen klimatischen und anbautechnischen Bedingungen stellen diese Rohstoffe die vielversprechendsten Optionen für eine Produktion von Ethanol dar. Andere nutzbare Kulturpflanzen wie Zuckerhirse, Gehaltsrüben, Topinambur und Zichorien erfüllen die genannten Kriterien (Anbauerfahrung, großtechnische Nutzung) nicht und kommen daher als Rohstoff für die Ethanolerzeugung derzeit nicht in Betracht.

Traditionell eine große Bedeutung für die Ethanolerzeugung in Deutschland hat die Kar- 
toffel. Rund $50 \%$ des von der Bundesmonopolverwaltung für Branntwein übernommenen Ethanols kommt aus kleinen landwirtschaftlichen Brennereien, die auf Kartoffelbasis arbeiten. Kartoffeln sind für die Ethanolerzeugung ein problematischer Rohstoff. Nachteilig sind vor allem die hohen Rohstoff-, Lager- und Verarbeitungskosten, die eingeschränkte Verwendbarkeit der Schlempe sowie der hohe Abwasseranfall. Kartoffeln spielen aufgrund dieser gravierenden Kostennachteile keine Rolle für die Bioethanolerzeugung.

Zuckerhaltige Rohstoffe spielen global betrachtet die dominierende Rolle bei der Ethanolerzeugung. ${ }^{11}$ Bei der Umwandlung von Sonnenenergie in Biomasse erbringt die Zuckerrübe auf die Fläche bezogen die höchste Leistung aller Nutzpflanzen, die in den gemäßigten Zonen angebaut werden. Dies ist auf ihre Fähigkeit zur intensiven Photosynthese auch bei relativ niedrigen Temperaturen zurückzuführen.

Zuckerrüben können direkt, in Form von Dick- oder Dünnsaft und über Melasse zu Ethanol verarbeitet werden. Zuckerrüben weisen zwar die höchste Flächenproduktivität auf, haben andererseits aber deutliche Nachteile im Hinblick auf Lager- und Logistikkosten. Zuckerrüben stellen relativ hohe Anforderungen an Böden, wodurch der Anbau regional begrenzt ist. Bislang werden nur rund $25 \%$ der für den Zuckerrübenanbau verwendbaren Fläche genutzt, so dass auch ein deutlicher Ausbau für eine Bioethanolerzeugung möglich wäre. Hinzu kommt, dass sich die Situation nach Auslaufen der derzeit geltenden Zuckermarktordnung ohnehin ändern dürfte. Die unsicheren Rahmenbedingungen sowie die hohen Lager- und Logistikkosten sind mit dafür verantwortlich, dass bislang keine der neuen Bioethanolanlagen $\mathrm{Zu}$ ckerrüben als Rohstoffbasis gewählt hat.

Der Getreideanbau zur Ethanolerzeugung zielt vor allem auf einen möglichst hohen Stärkegehalt ab. Weizen weist einen hohen Stärkegehalt und eine gute Kornausbildung auf, ist allerdings ein relativ teurer Rohstoff, der zudem hohe Ansprüche an die Böden stellt. Dagegen können Roggen und Triticale auch auf ertragsschwachen Böden angebaut werden. Diese Getreidearten erreichen aber im Vergleich zu Weizen eine geringere Flächenproduktivität. Bei Roggen ist die Verarbeitung zu Ethanol etwas teurer, da viskositätssenkende
Enzyme zur Verhinderung der Verschleimung im Konversionsprozess beigegeben werden müssen und ein höherer Dampfbedarf aufgrund schlechterer Möglichkeiten der Schlemperückführung erforderlich ist. Die Verfügbarkeit von Roggen am Markt ist derzeit abhängig von der geltenden Marktordnung.

Die Ethanolherstellung auf Maisbasis spielt bislang in Deutschland keine Rolle. ${ }^{12}$ Zukünftig kann sich dies ändern, wenn neue Sorten zugelassen werden, bei denen der Mais noch auf dem Feld trocknen kann, bevor er geerntet wird. Dadurch wird die sonst erforderliche aufwändige Trocknung vor der Verarbeitung eingespart. Damit kann Mais zu einem attraktiven Rohstoff für die Ethanolerzeugung in Deutschland werden.

Insgesamt kann festgestellt werden, dass die in Deutschland zur Verfügung stehenden landwirtschaftlichen Anbauflächen grundsätzlich keinen limitierenden Faktor für die Erreichung der genannten Beimischungsziele darstellen, allerdings müssen hier möglicherweise auftretende Nutzungskonkurrenzen berücksichtigt werden. ${ }^{13}$ Verwendet man die derzeitigen Getreide- und Zuckerexporte sowie die noch nicht für den Rapsanbau verwendeten Stilllegungsflächen, dann können knapp 8 Mio. $\mathrm{m}^{3}$ Bioethanol hergestellt werden (vgl. Tab. 2 nächste Seite).

Für die EU-15 wird das Potenzial an Biokraftstoffen aus Energiepflanzen, Abfall- und Reststoffen auf $15 \%$ des gesamten Kraftstoffverbrauchs geschätzt (BMVBW 2001).

Neben der Verwendung traditioneller landwirtschaftlicher Produkte wird zunehmend die Verarbeitung von lignozellulosehaltigen Rohstoffen diskutiert, bei denen Zellulose, Lignin und Hemizellulose für die Ethanolerzeugung genutzt werden. $\mathrm{Zu}$ diesen Pflanzen zählen etwa schnellwachsende Baumarten (z. B. Pappeln, Weiden), Miscanthus und Rutenhirse. Mittel- bis langfristig sind zellulosehaltige Rohstoffe vielversprechend, da sie zum einen in sehr großer Menge und zum anderen wahrscheinlich zu niedrigen Kosten zur Verfügung stehen können. Bislang bestehen allerdings erhebliche technische Schwierigkeiten, Ethanol auf dem Wege der Fermentation aus zellulosehaltigen Rohstoffen zu gewinnen; zudem sind die Konversionskosten noch deutlich zu hoch, um wirtschaftlich Ethanol produzieren zu können. Schließlich bestehen ungelöste Prob- 
Tab. 2: Potenzial für die Bioethanolproduktion in Deutschland

\begin{tabular}{|c|c|c|c|}
\hline & Produktion / verfügbare Fläche & Potential für Bioethanol & $\begin{array}{c}\text { Potenzielles } \\
\text { Bioethanol- } \\
\text { Produktionsvolumen }\end{array}$ \\
\hline Getreide & $\begin{array}{l}\text { - Erntevolumen ca. } 45 \text { Mio. t } \\
\text { p. a. in Deutschland } \\
\text { - Anbaufläche beträgt etwa } 6,9 \\
\text { Mio. ha, entspricht etwa } 60 \% \\
\text { der Ackerfläche }\end{array}$ & $\begin{array}{l}\text { - knapp } 15 \text { Mio. t Getreide- } \\
\text { exporte (Durchschnitt der } \\
\text { letzten } 5 \text { Jahre) }\end{array}$ & - $\quad$ 5,3 Mio. $\mathrm{m}^{3}$ \\
\hline Zuckerrüben & $\begin{array}{l}\text { - rund 3,8 Mio. t Zuckerproduk- } \\
\text { tion p. a. } \\
\text { - Anbaufläche beträgt etwa } \\
450.000 \text { ha }\end{array}$ & $\begin{array}{l}\text { rund 1,3 Mio. t Zuckerex- } \\
\text { porte p. a. (Durchschnitt } \\
\text { der letzten } 5 \text { Jahre) }\end{array}$ & - $\quad$ 0,8 Mio. $\mathrm{m}^{3}$ \\
\hline $\begin{array}{l}\text { Stilllegungs- } \\
\text { flächen }\end{array}$ & $\begin{array}{l}\text { - } 1,2 \text { Mio. ha Stilllegungsflächen } \\
\text { - ca. } 300.000 \text { ha Nutzung für } \\
\text { Rapsanbau }\end{array}$ & $\begin{array}{l}\text { - } 800.000 \text { ha für Anbau von } \\
\text { Rohstoffen für die Bio- } \\
\text { ethanolerzeugung (Kon- } \\
\text { kurrenz zu anderen Nut- } \\
\text { zungsoptionen muss be- } \\
\text { rücksichtigt werden) }\end{array}$ & - 1,8 Mio. $\mathrm{m}^{3}$ \\
\hline
\end{tabular}

Quelle: persönliche Mitteilung der Südzucker AG

leme bei der Aufbereitung des Abwassers. Dieser Verarbeitungsansatz kommt daher für ein Bioethanolprogramm in Deutschland derzeit nicht in Betracht, kann allerdings für die $\mathrm{Zu}-$ kunft eine interessante Option darstellen.

\section{Bioethanol in Brasilien}

Wie aus Abbildung 2 hervorgeht, hat die weltweite Ethanolproduktion in den letzten Jahren stetig zugenommen und lag 2004 bei rd. 40 Mio. $\mathrm{m}^{3}$. Hinsichtlich der regionalen Verteilung ist hierbei Südamerika zwar dominierend, aktuell haben aber die USA Brasilien als bisher weltgrößten Ethanolhersteller überholt. Im Gegensatz dazu verfügt Brasilien weltweit aber immer noch über die deutlich kostengünstigste Ethanolproduktion; deshalb soll hierauf näher eingegangen werden. In Brasilien liegen die Vollkosten der Ethanolproduktion bei 200 bis 250 US\$ pro $\mathrm{m}^{3}$ Ethanol - bei einem Dollarkurs von 1,23 entspricht dies 163 bis $203 €$ pro $\mathrm{m}^{3}$. In Europa liegen die Vollkosten bei konventionellen Anlagen derzeit zwischen 450 und $500 €$ pro $\mathrm{m}^{3}$. Aufgrund des relativ geringen mineralischen Düngemittelbedarfs beim Anbau des Zuckerrohrs sowie des Energieüberschusses im Konversionsprozess - durch die energetische Nutzung der Bagasse - ist die Energiebilanz sehr günstig. Entsprechend hoch sind die Einsparungen an Treibhausgasen.
Abb. 2: Entwicklung der weltweiten Ethanolproduktion und deren Verteilung

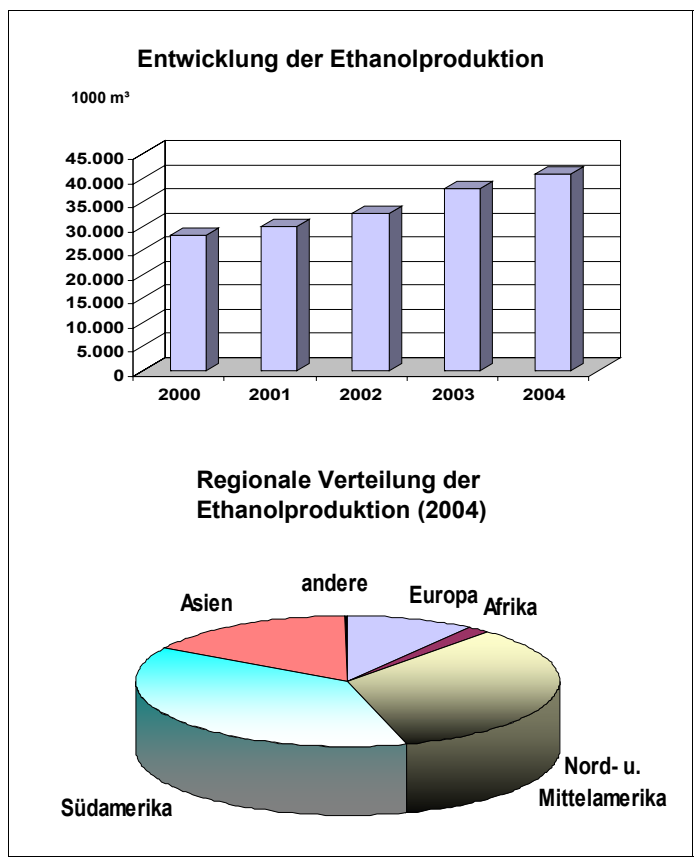

Quelle: Zusammenstellung nach F.O. Licht (2004)

Brasilien verfügt über die weltweit größten Anbauflächen für Zuckerrohr. Die Zuckerrohrproduktion ist in der Centre-South-Region konzentriert, insbesondere in den Staaten São Paulo und Paraná (85\% des gesamten Anbaus). Der Rest 
wird im Nordosten Brasiliens angebaut, allerdings unter ungünstigeren Bedingungen.

Der Zuckerrohranbau Brasiliens nimmt gegenwärtig einen relativ kleinen Teil der landwirtschaftlichen Nutzfläche ein. Das brasilianische Landwirtschaftsministerium geht von einer landwirtschaftlichen Nutzfläche von 320 Millionen Hektar aus. Zurzeit werden davon etwa 53 Millionen Hektar in Anspruch genommen, für den Zuckerrohranbau werden 5,6 Millionen Hektar genutzt (vgl. Abb. 3).

Heute wird in Brasilien jeweils etwa die Hälfte des Zuckerrohrs für die Zucker- und Ethanolproduktion verwendet. Im Jahr 2003 wurden in Brasilien 350 Mio. $t$ Zuckerrohr geerntet. Große Schwankungen in den Erntemengen sind vor allem auf die Wetterverhältnisse zurückzuführen. Es existieren etwa 400 Ethanol-Konversionsanlagen mit einer Gesamtkapazität von derzeit etwa 18 Mio. $\mathrm{m}^{3}$ p. a. Diese Anlagen verarbeiten etwa zwei Drittel der Zuckerrohr-Biomasse. Neue Technologien sind in der Entwicklung, um auch aus dem sog. Zuckerrohrstroh (Blattmasse), das heute überwiegend auf den Feldern abgebrannt wird,
Ethanol zu gewinnen. Ein weiterer Anreiz zur Optimierung wurde durch das neue brasilianische Energieeinspeisegesetz gesetzt. Überschüssige Bagasse von den Zucker- und Ethanolfabriken kann verstromt und in das öffentliche Stromnetz eingespeist werden.

Seit der Einführung des Proálcool-Programms 1975 wird in Brasilien dem Benzin Ethanol beigemischt. Heute gibt es im brasilianischen Kraftstoffmarkt nur Benzin mit einer Beimischung von $25 \%$ Ethanol (E-25) und reines Ethanol (E-100). Der jährliche Verbrauch liegt seit Ende der 1980er Jahre bei rund 12 Mio. $\mathrm{m}^{3}$, wobei der Verbrauch von Ethanol als Reinkraftstoff von 1997 bis 2002 rückläufig war, in den letzten Jahren aber wieder anstieg. Derzeit werden rund 14 Mio. $\mathrm{m}^{3}$ Ethanol im Inlandsmarkt als E-100 oder E-25 abgesetzt. Ethanolfreies Benzin ist im brasilianischen Markt nicht vorhanden.

Für diese zwei Kraftstofftypen existieren auf dem brasilianischen Markt heute drei verschiedene Fahrzeugtypen. Für das sog. Gasohol (E-25) werden normale Ottomotoren geringfügig modifiziert. Die in Brasilien angebotenen

\section{Abb. 3: Brasilien - Entwicklung der landwirtschaftlichen Nutzfläche, Zuckerrohranbaufläche und Zuckerrohr- bzw. Ethanolproduktion}

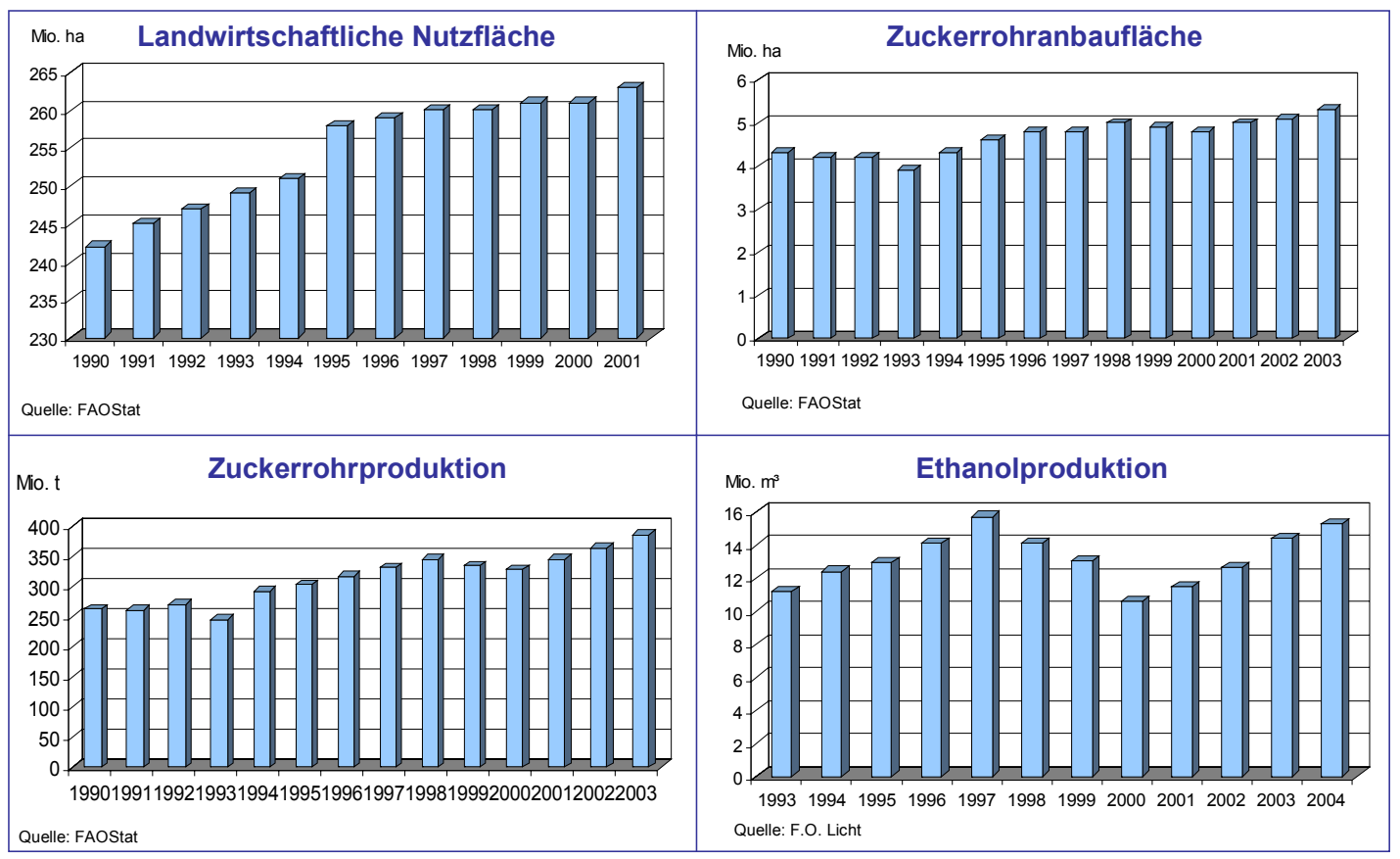

Quelle: Schmitz 2005 
Fahrzeuge verarbeiten den ethanolhaltigen Kraftstoff problemlos. Selbst aus Deutschland importierte Luxusfahrzeuge haben mit dem brasilianischen Gasohol keine Probleme. Die Beimischung von Ethanol führt sogar zu einer verbesserten Motorleistung. Die reinen Ethanolfahrzeuge, die mit E-100 betankt werden, sind stärker modifiziert. Seit 2003 werden auch FFVs auf dem Markt angeboten, mit denen sowohl E-25 als auch E-100 getankt werden kann. Im Jahr 2004 waren etwa $30 \%$ der Neuzulassungen FFVs, im Jahr 2010 soll bereits ein Viertel der gesamten Fahrzeugflotte aus FFVs bestehen. Der Erfolg der FFVs im Markt hat sich in 2005 noch weiter verstärkt. Die Mineralölindustrie erwartet aus diesem Grunde eine deutliche Ausweitung der Binnennachfrage nach Ethanol.

Angesichts der erwarteten rasanten Steigerung der Ethanolnachfrage stellt sich die Frage, wie die Investitionen in die Ethanolindustrie und die Erschließung neuer Anbauflächen in relativ kurzer Zeit realisiert werden können. Für den internationalen Markt bedeutet dies unter Umständen, dass angesichts der starken Binnennachfrage nur ein geringes Angebot aus Brasilien zur Verfügung stehen könnte. Zudem ist die Infrastruktur unzureichend, was sich u. a. in hohen Logistikkosten und Risiken niederschlägt. Darüber hinaus ist zu erwarten, dass steigende Ethanolpreise in Brasilien auch den Weltmarktpreis von Ethanol nach oben ziehen werden. Dieser Prozess würde umso stärker ausfallen, je länger der Trend steigender Ölpreise anhält.

Als große Chance für die brasilianische Ethanolindustrie wird die weltweit stark wachsende Nachfrage nach Bioethanol betrachtet. In einer wachsenden Zahl von Ländern in Europa und Asien wird die Verwendung von Biokraftstoffkomponenten forciert. Länder wie Japan und Korea haben erheblichen Importbedarf. Auch Exporte in die USA spielen eine wachsende Rolle. Allerdings ist auch bekannt, dass die Preise für brasilianisches Ethanol in der Vergangenheit immer sehr volatil waren, bedingt durch schwankende Nachfrage und unterschiedliche Zuckerpreise. Vor dem Hintergrund des erwarteten starken Anstiegs der Nachfrage sowohl im Heimatmarkt als auch in Nordamerika, Asien und Europa expandiert derzeit die brasilianische Ethanolproduktion deutlich.

\section{Energie- und Treibhausgasbilanzen}

Der Einsatz von Bioethanol als erneuerbarer Kraftstoff war aus klimapolitischer Sicht lange Zeit nicht unumstritten. Gegner argumentieren mit ungünstigen Treibhausgasbilanzen aufgrund aufwändiger Umwandlungsprozesse und geringer Energieausbeuten. Vorliegende Studien kommen in Abhängigkeit von den getroffenen Annahmen zu unterschiedlichen Ergebnissen. Vielen wissenschaftlichen Studien ist gemeinsam, dass jüngere Entwicklungen bei der Optimierung von Verfahren und Technologien in Brennereien und in der Landwirtschaft sowie Gutschriften für anfallende Kuppelprodukte nicht ausreichend berücksichtigt sind. In jüngster Vergangenheit erschienene Studien bestätigen dies (vgl. Quirin et al. 2004; Schmitz 2005). Allerdings ist die Varianz der Ergebnisse sehr groß. Sie resultiert vor allem aus unterschiedlichen Ansätzen zur ökonomischen und ökologischen Bewertung der Kuppelprodukte, unterschiedlichen Verfahren in der landwirtschaftlichen Produktion und in der Konversion sowie aus unterschiedlichen System- bzw. Bilanzgrenzen. ${ }^{14}$

Entsprechend zeigen auch aktuelle Studien bei den Energiegewinnen erhebliche Spannbreiten von 4,3 $\mathrm{MJ} /$ Liter Ethanol bis 26,4 MJ / Liter (vgl. Schmitz 2005). Die überwiegende Zahl der Studien geht von Energiegewinnen zwischen 8 und $15 \mathrm{MJ} /$ Liter aus. Bei den Treibhausgasbilanzen reicht die Spanne der Einsparungen von $0,5 \mathrm{~kg}$ bei der Bioethanolproduktion aus Weizen bis $2,24 \mathrm{~kg}$ $\mathrm{CO}_{2}$-Äquivalente / Liter Ethanol bei der Produktion aus Zuckerrohr in Brasilien (vgl. Macedo et al. 2004). Für Deutschland liegt der beste Wert bei $1,5 \mathrm{~kg} \mathrm{CO}_{2}$-Äquivalente / Liter Ethanol bei der Produktion aus Zuckerrüben.

Die Konversion bietet das größte Optimierungspotential in der gesamten Erzeugungskette - beginnend mit der landwirtschaftlichen Produktion bis zur Verwendung des Ethanols im Kraftstoffsektor. Unterschiedliche Produktionskonzepte reichen von traditionellen, bereits seit vielen Jahren im Betrieb befindlichen Anlagen bis hin zu Konzepten, die noch im Entwicklungsstadium sind. Auch unterschiedliche Betriebsgrößen sind $\mathrm{zu}$ berücksichtigen. Die für eine Bioethanolerzeugung in Europa in Frage kommenden Rohstoffe sind Weizen, 
Tab. 3: Nettoenergiegewinn und Treibhausgasreduzierung bei verschiedenen Rohstoff- und Energieoptionen der Ethanolgewinnung

\begin{tabular}{|l|c|c|}
\hline Rohstoff / Energieträger & $\begin{array}{c}\text { Netto-Energiegewinn (Output abzgl. } \\
\text { fossilem Input) (MJ / l Ethanol) }\end{array}$ & $\begin{array}{c}\text { Treibhausgasreduzierung } \\
\left(\mathrm{kg} \mathrm{CO}_{2} \text {-̈̈quivalent / l Ethanol }\right.\end{array}$ \\
\hline Melasse / Heizöl S & 6,4 & 0,8 \\
\hline Rübensaft / Braunkohle & 6,6 & 0,3 \\
\hline C-Stärke + Melasse / Erdgas & 8,9 & 1,1 \\
\hline Getreide / Erdgas & 6,6 & 0,7 \\
\hline Getreide / Müll & 14,5 & 1,1 \\
\hline Getreide / Biogas & $19,2-21,3$ & $1,1-1,5$ \\
\hline Stroh / Biogas & $15,7-20,1$ & $1,6-1,8$ \\
\hline
\end{tabular}

*) 11 Ethanol $\triangleq$ 0,65 1 Benzin bzgl. des Heizwerts; Heizwert Ethanol: 21,17 MJ / 1; Heizwert Benzin: 32,45 MJ / 1

Quelle: Schmitz 2005

Roggen, Triticale, Melasse, Zuckerrüben und Stroh. Ein weiteres wichtiges Differenzierungsmerkmal, das erhebliche Auswirkungen auf die Bilanzierungsergebnisse hat, ist der für die Konversion eingesetzte Brennstoff. Er reicht von Biogas, Erdgas und Müll über Braunkohle bis hin zu schwerem Heizöl. Die Verwendung nicht-fossiler Prozessenergie (im Konversionsprozess gewonnenes Methangas, Energie aus Müllverbrennung) entlastet die Treibhausgasbilanz beträchtlich.

Die Erzeugung von Bioethanol aus Stroh verzeichnet aufgrund des verwendeten Ausgangsstoffs und der geringen erforderlichen fossilen Prozessenergie die geringsten Klimagasemissionen. Entsprechend hoch sind die Treibhausgaseinsparungen: Sie liegen zwischen 1,89 und $2,15 \mathrm{~kg} \mathrm{CO}_{2}$-Äquivalenten pro Liter Ethanol. Auch die Erzeugung von Ethanol aus Getreide, gekoppelt mit einer Biogas-Anlage, führt zu geringen Klimagasemissionen. Bei in Betrieb befindlichen Anlagen schneiden die Konversionspfade der Verarbeitung von minderwertiger Stärke (C-Stärke) und Getreide, verbunden mit der Verwendung nicht-fossiler Prozessenergie, am besten ab. Die Treibhausgasreduzierung liegt dabei bei 1,39 bzw. $1,49 \mathrm{~kg}$ $\mathrm{CO}_{2}$-Äquivalenten. Die größten Klimagasemissionen entstehen bei der Produktion von Ethanol aus Rübensaft und der Verwendung von Braunkohle als Energieträger. Tab. 3 gibt einen zusammenfassenden Überblick über die relative
Vorzüglichkeit der verschiedenen Rohstoff- und Energieoptionen der Ethanolproduktion.

\section{Schlussbemerkungen}

Bislang beschränkt sich die BioethanolNachfrage in Deutschland weitgehend auf die Versorgung des ETBE-Marktes. Die Perspektiven für die Bioethanolerzeugung werden wesentlich durch politische Parameter und weniger durch Konsumentenpräferenzen beeinflusst (vgl. Abb. 4 nächste Seite).

Die Automobilindustrie spricht sich mittlerweile für eine verstärkte Verwendung von Biokraftstoffen aus (siehe Beitrag von Seyfried in diesem Schwerpunkt). DaimlerChrysler hat sich beispielsweise aus Anlass des vierten Umweltforums des Unternehmens in Magdeburg verpflichtet, Fahrzeuge herzustellen, die mit einem Anteil von $10 \%$ Biokraftstoffen betrieben werden können. Gleichzeitig will das Unternehmen die Entwicklung entsprechender Kraftstoffstandards gemeinsam mit anderen Unternehmen und Institutionen vorantreiben. DaimlerChrysler wie auch andere Automobilhersteller erhoffen sich aus der verstärkten Verwendung von Biokraftstoffen Gutschriften bei den $\mathrm{CO}_{2}$-Emissionswerten ihrer Fahrzeuge. Dies dürfte mittelfristig zu einer erheblichen Ausweitung der Nachfrage nach Bioethanol führen. Es ist wahrscheinlich, dass in Deutschland bzw. in der EU ein E-10-Kraftstoff genauso Standard 


\section{Abb. 4: Bestimmende Parameter der Ethanolproduktion in Deutschland}

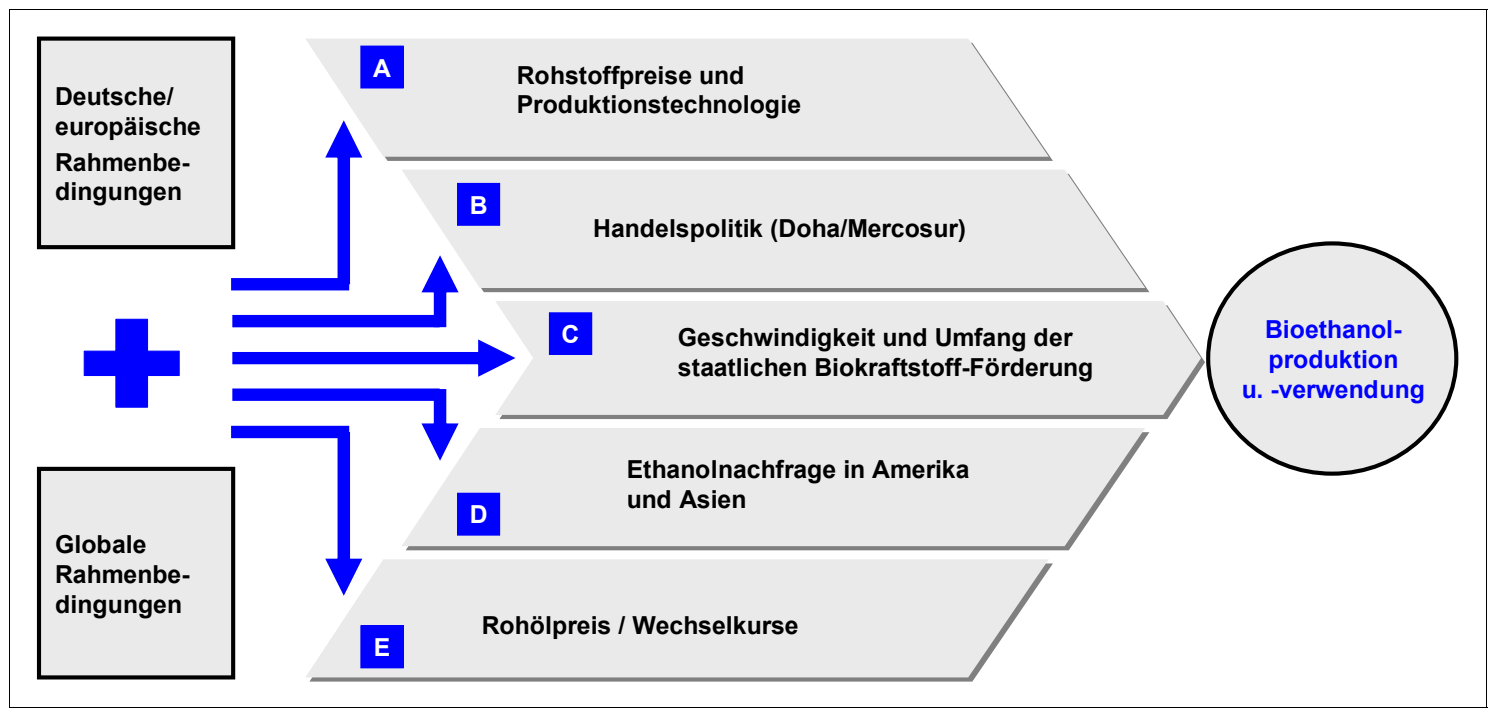

wird wie in den USA. Es ist unwahrscheinlich, dass die Mineralölindustrie dauerhaft einer Etherstrategie den Vorzug gibt und auf die direkte Beimischung von Ethanol verzichtet.

Nicht förderlich für die weitere Entwicklung des Biokraftstoffmarktes ist die Situation in der EU-25. Dieser Markt gleicht einem Flickenteppich. In den verschiedenen Ländern werden verschiedene Systeme zur Förderung von Biokraftstoffen implementiert, die in aller Regel heimische Produzenten massiv bevorzugen. In Frankreich oder Spanien kommen z. B. nur Biokraftstoffe von so genannten lizenzierten heimischen Herstellern in den Genuss einer steuerlichen Förderung. Ausländische Wettbewerber haben dagegen keine Chance. Gleichzeitig sind die so in ihren Heimatländern geförderten Unternehmen als Exporteure in den „freien“ EU-Märkten Deutschland und Schweden aktiv. Die EU-Kommission sollte hier ihre originäre Aufgabe wahrnehmen und gleiche Bedingungen für einen funktionierenden Wettbewerbsmarkt schaffen.

\section{Anmerkungen}

1) ETBE steht für Ethyl-Tertiär-Butyl-Ether. Es handelt sich um einen Oktanzahlverbesserer, der aus Bioethanol und Isobuten hergestellt wird.

2) TAEE steht für Tertiär-Amyl-Ethyl-Ether.

3) Die Zielwerte beziehen sich dabei auf den substituierten Energiegehalt (unterer Heizwert).
4) FAEE steht für Fatty Acid Ethyl Ester. Es entspricht FAME, allerdings wird bei der Produktion nicht Methanol sondern Ethanol eingesetzt.

5) $1 \mathrm{~m}^{3}$ Bioethanol besitzt ein Gewicht von $0,79 \mathrm{t}$ (Anm. d. Red.).

6) Bei Mischungen von Ottokraftstoff mit Ethanol kommt es zu einer Veränderung des Dampfdrucks. Der Dampfdruck resultiert aus den Kraftstoffkomponenten, die bei einer definierten Temperatur in einem geschlossenen Behälter aus der Flüssigphase in die Dampfphase übergehen. Bei der Beimischung von Ethanol treten hier Dampfdruckanomalien auf. Ethanol weist einen niedrigeren Dampfdruck als Ottokraftstoff auf, so dass bei Zumischung der Dampfdruck des Gemisches abfallen sollte. Dies ist allerdings bei niedrigen Ethanolkonzentrationen nicht der Fall. Bei höheren Ethanolgehalten nimmt die Dampfdruckanomalie ab (vgl. Schmitz 2003).

7) Bei dem in den neuen Bundesländern in 2005 durchgeführten Direktbeimischungs-Feldversuch wurden nach Aussagen von Vertretern der Mineralölindustrie erhebliche Wasserprobleme festgestellt.

8) Dem Autor sind zahlreiche Projekte in unterschiedlichen Entwicklungsstadien bekannt.

9) DDGS steht für „Dried Distillers Grains and Solubles“. Dies ist ein eiweißreiches Futtermittel, das durch die Trocknung der bei der Ethanolproduktion anfallenden Schlempe gewonnen wird, wenn als Rohstoff Getreide (z. B. Mais, Weizen) zum Einsatz kommt.

10) Flexible Fuel Vehicles sind an den Kraftstoff anpassungsfähige Fahrzeuge, deren Motoren sowohl mit reinem Benzin als auch mit verschiede- 
nen Mischungsanteilen (bis 85 Vol.-\%) von Ethanol im Benzin zurecht kommen.

11) Rund drei Fünftel der globalen Produktion erfolgt auf der Basis von Zuckerrohr und $\mathrm{Zu}$ ckerrüben, während die Ethanolerzeugung auf Basis von Getreide nur ein Drittel und die Synthese-Ethanol-Erzeugung weniger als ein Zehntel ausmacht (vgl. F.O. Licht 2003, S.1).

12) In den USA wird überwiegend Mais als Rohstoff für die Bioethanolproduktion eingesetzt. Im Süden Frankreichs soll eine erste Mais verarbeitende Bioethanolanlage entstehen.

13) Zur Verfügbarkeit von Biomasse für die Herstellung von Biokraftstoffen vgl. z. B. die aktuelle Studie von Leible et al. (2005).

14) Eine ausführliche Darstellung zu diesem Thema findet sich bei Schmitz (2005).

\section{Literatur}

BMVBW - Bundesministerium für Verkehr, Bau- und Wohnungswesen 2001: Verkehrswirtschaftliche Energiestrategie (VES) - eine gemeinsame Initiative von Politik und Wirtschaft.; http://www.bmvbw.de/ Mobilität-.643.2458/.htm (Zugang: 2004)

BMVEL - Bundesministerium für Ernährung, Landwirtschaft und Verbraucherschutz, 2005: Biokraftstoffe. Strategie für Mobilität von morgen. Berlin

Deutscher Bundestag, 2005: Unterrichtung durch die Bundesregierung. Bericht zur Steuerbegünstigung für Biokraft- und Bioheizstoffe. Drucksache $15 / 5816,21.06 .2005$. Berlin

F.O. Licht, 2003: World Ethanol Markets. The Outlook to 2012. London: Agra Europe

F.O. Licht, 2004: World Ethanol and Biofuels Report. London: Agra Europe

FAO - Food and Agriculture Organization of the United Nations, 2005: FAOStat data; http://faostat.fao.org/faostat (Zugang: 2005)

FNR - Fachagentur Nachwachsende Rohstoffe e.V. (Hrsg.), 2005: Basisdaten Biokraftstoffe. Stand: Januar 2005. Gülzow: FNR

IEA - International Energy Agency, 2004: Biofuels for Transport - An International Perspective. Paris: IEA

Leible, L.; Kälber, S.; Kappler, G., 2005: Entwicklungen von Szenarien über die Bereitstellung von land- und forstwirtschaftlicher Biomasse in zwei baden-württembergischen Regionen zur Herstellung von synthetischen Kraftstoffen. Mengenszenarien zur Biomassebereitstellung. Studie im Auftrag der DaimlerChrysler AG. Abschlussbericht, Juni 2005. Erstellt durch: Forschungszentrum Karlsruhe $\mathrm{GmbH}$, Institut für Technikfolgenabschätzung und
Systemanalyse (ITAS); http://www.itas.fzk.de/deu/ lit/2005/leua05a.pdf (Zugang: 2006)

Macedo, I. de Carvalho; Lima Verde Leal, M.R.; Azevedo Ramos da Silva, J.E., 2004: Assessment of greenhouse gas emissions in the production and use of fuel ethanol in Brazil. São Paulo: Government of the State of São Paulo, Secretariat of the Environment

Quirin, M., Gärtner, S., Pehnt, M., Reinhardt, G., 2004: $\mathrm{CO}_{2}$-neutrale Wege zukünftiger Mobilität durch Biokraftstoffe. Eine Bestandsaufnahme. Endbericht. Ifeu, Union zur Förderung von Oel- und Proteinpflanzen (UFOP) und Forschungsvereinigung Verbrennungskraftmaschinen (FVV). Heidelberg, Mai 2004

Schmitz, N. (Hrsg.), 2003: Bioethanol in Deutschland. Verwendung von Ethanol und Methanol aus nachwachsenden Rohstoffen im chemisch-technischen und im Kraftstoffsektor unter besonderer Berücksichtigung von Agraralkohol. Schriftenreihe „Nachwachsende Rohstoffe“, Band 21. Münster: Landwirtschaftsverlag

Schmitz, N. (Hrsg.), 2005: Innovationen bei der Bioethanolerzeugung und ihre Auswirkungen auf Energie- und Treibhausgasbilanzen. Neue Verfahren, Optimierungspotenziale, internationale Erfahrungen und Marktentwicklungen. Schriftenreihe „Nachwachsende Rohstoffe“, Band 26. Münster: Landwirtschaftsverlag

\section{Kontakt}

Dr. Norbert Schmitz

Meó Consulting Team

Weissenburgstr. 53, 50670 Köln

Tel.: +49 (0) 221 / 9727232

E-Mail:schmitz@meo-consulting.com 